\title{
SUR UN PROTOPHYTE PARASITE INTESTINAL
}

\section{DE POISSON MARIN}

\author{
Par G. LAVIER
}

Le curieux entophyte qui fait l'objet de cette note a été rencontré au cours d'un séjour en avril 1935 au Laboratoire Arago à Banyuls, alors que je recherchais systématiquement des flagellés intestinaux de poissons marins. Je l'ai observé chez les deux individus de Mugil chelo Cuv. et Val. que j'ai eus à ma disposition et chez eux seulement. Au cours de l'examen à frais du contenu intestinal de ces poissons, j'avais noté sa présence, mais sans pouvoir juger ni de sa nature ni même de sa qualité de parasite ; comme le mucus, dans les deux cas contenait de nombreux Hexamita mugilis (cf. Lavier, 1936), j'effectuai immédiatement un certain nombre de frottis que je fixai au liquide de Bouin et colorai plus tard par l'hématoxyline ferrique. En recherchant les flagellés dans les préparations je retrouvai mon protophyte et pus alors en reconnaître la nature parasitaire. Ces circonstances expliquent que, mon attention n'ayant pas été immédiatement attirée sur lui, je n'aie pas procédé à des colorations vitales et à des fixations spéciales qui eussent permis d'en préciser la cytologie ; de là viennent les lacunes qu'on trouvera malheureusement dans cette étude et qu'un matériel nouveau seul permettrait de combler. Mais pour incomplètes que soient les données que m'ont fournies mes préparations, elles montrent que cet organisme occupe une place spéciale parmi les Schizophytes et soulève un intéressant problème d'affinités.

Morphologie et évolution. - A frais, il se présentait sous forme de filaments claviformes ou en croissants très allongés, entourés d'une gaine hyaline mince mais facile à observer (fig. 2) et formés d'articles successifs plus ou moins réfringents, de largeur variable suivant leur position mais plus larges que longs. La taille de ces filaments était fort variable; quand ils étaient courts, on pouvait nettement constater leur aplatissement; dans des filaments plus longs, la mise au point du microscope montrait que vers l'extrémité distale, l'article s'incurvait dans le sens transversal, pliant ses deux bords latéraux, transformé ainsi en une sorte de gouttière

Annales de Parasitologie, T. XVI, $\mathrm{N}^{\circ} 3 .-1^{\text {er }}$ mai 1938, p. 259-264. 
très aplatie, comme si, devenu trop large pour un contenant rigide, il n'avait que cette manière de prendre toute son expansion latérale. Plusieurs de ces filaments présentaient des mouvements lents de latéralité qui paraissaient bien être actifs. Je n'ai pas alors observé la fixation du protophyte ; pour les raisons que j'ai dites plus haut, d'ailleurs, mon observation à frais a été courte.

Dans les frottis fixés et colorés, les filaments sont nombreux, mesurant de 8 jusqu'à $150 \mu$ environ de longueur. Sur des placards épithéliaux restés collés à la lame, on peut observer leur fixation qui se fait souvent par véritables touffes à la surface des cellules intestinales mettant ainsi hors de doute leur nature parasitaire ou tout au moins saprophytique (fig. 1). L'épithélium ne manifeste d'ailleurs aucune altération apparente.

Le cône de fixation qui reste incolore, mais dont le contour apparaît en négatif dans le frottis, a sa base appliquée à l'origine du filament; sa pointe plonge probablement dans le plateau épithélial comme c'est le cas chez l'Anisomitus denisi Grassé du canard, mais l'absence de coupes ne me permet pas d'être affirmatif à cet égard. La gaine facilement visible à frais n'est plus observable avec certitude ; sans doute a-t-elle souffert du fixateur. Quant aux articles qui composent le filament, leur aspect varie avec l'âge et, à ce point de vue, grands et petits filaments ou extrémité proximale et extrémité distale du même peuvent présenter de notables différences.

Les filaments jeunes montrent à leur. extrémité proximale des articles très courts pouvant mesurer moins d'un $\mu$ de longueur sur $1 \mu$ en moyenne de largeur, mais tandis que la largeur s'accroît ensuite rapidement pour atteindre environ $3 \mu$ vers le $10^{\circ}-12^{\circ}$ article, la longueur ne croît que lentement ; elle n'est d'ailleurs jamais guère supérieure à $2 \mu$. Une membrane très facile à observer revêt extérieurement l'ensemble des articles. Ceux-ci présentent des affinités chromatiques très variables ; çà et là des cloisons transversales sidérophiles montrant le mode d'accroissement du protophyte sont mises en évidence; l'aspect rappelle alors beaucoup celui des Oscillatoriées. Le contenu des articles paraît finement granuleux et homogène, mais il faut bien dire que la technique des frottis n'est pas de celles dont on peut attendre de grandes précisions cytologiques, de sorte qu'il est impossible de dire s'il y a un corps central ou une chromatine diffuse (fig. $3,4,6$ ).

Par la suite, l'article continuant à augmenter transversalement sans que, pour autant, le filament s'élargisse, se replie sur ses deux bords latéraux; l'examen soigné des préparations est en 

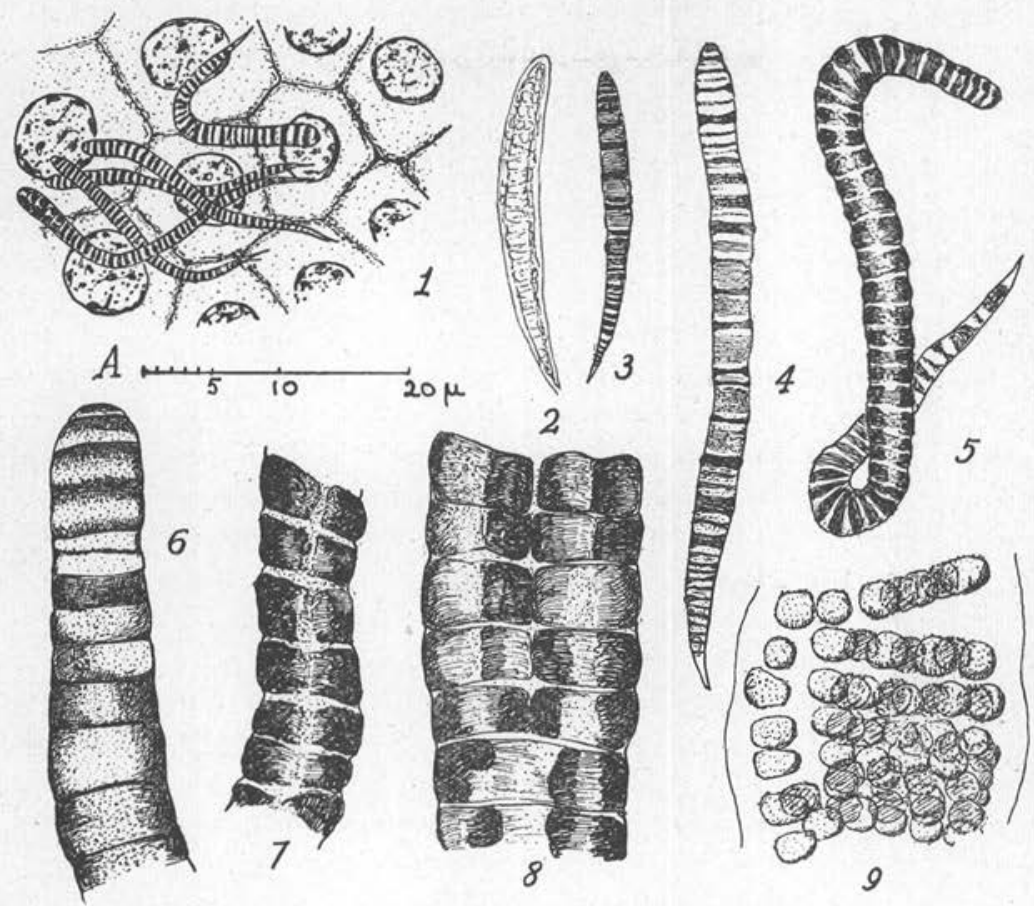

9

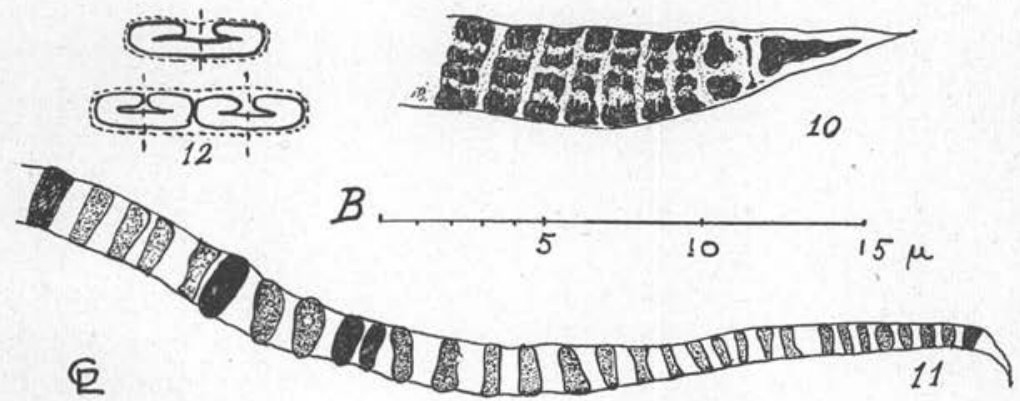

Figure. - Anacamptothrix intestinalis n. g., n. sp. de Mugil chelo. 1, placard épithélial avec filaments jeunes fixés à la surface ; 2 , un filament vu à frais (croquis) ; $3,4,5$, divers filaments ; 6 , extrémité distale d'un filament encore relativement jeune; 7 , portion d'un filament déjà âgé où les articles se replient en gouttière ; 8 , portion d'un filament âgé : division des articles et repli de chacune des moitiés ; 9 , conidies ; 10 , extrémité proximale d'un filament avec division des articles ; 11, filament à articles très espacés ; 12, schéma montrant en haut le repli de l'article, en bas le repli des deux moitiés après division. $1,3,4$, 5 et 11 à l'échelle $\mathrm{A} ; 6,7,8,9,10$ à l'échelle $\mathrm{B}$. 
cela bien d'accord avec les renseignements obtenus sur le frais ; on a un article simple incurvé en gouttière et pas, ou du moins pas encore de division dans le sens axial comme on pourrait le croire à première vue. Le même article se présente ainsi sur deux plans (voir schéma 12); le filament n'a plus alors l'aspect de ruban plat auquel les hasards du frottis peuvent imprimer des plis rigides à angles aigus; son épaisseur accrue le rend sinueux (fig. 5). Nombreux sont les filaments où l'on voit cette transformation se faire à partir d'un nombre d'ailleurs assez variable d'articles.

Plus loin dans le filament, on pourra voir la division de l'article incurvé se réaliser véritablement au niveau de l'axe longitudinal, et l'accroissement dans le sens transversal continuant toujours, les deux régions qui se sont séparées glissent l'une contre l'autre en se repliant et l'on a alors deux gouttières accolées, situées au même niveau (fig. 8,12 ). Tout à l'extrémité des vieux filaments, ce processus de division des articles va aboutir à la formation de spores sphéroïdales mesurant à peine plus d'un $\mu$ de diamètre (fig. 9). Il semble que chaque article en fournisse deux rangées de 8 à 12 environ réparties sur deux plans. Ce sont des exospores provenant de la fragmentation du thalle par division, mais à proprement parler suivant deux dimensions seulement: transversale et sagittale puisque la couche profonde ne dérive pas de la superficielle par division mais par repli seulement. Ces spores ne paraissent pas posséder de parois résistantes; il est vraisemblable que, libérées par rupture de la membrane générale du filament et de la gaine, elles sont susceptibles de reproduire de nouveaux filaments.

Peut-être y a-t-il d'autres modes de reproduction ; j'ai rencontré en effet quelques filaments (fig. 11) dont les articles très éloignés les uns des autres avaient une tendance nette à condenser leur cytoplasme et il est permis de se demander si de tels éléments libérés ne seraient pas susceptibles de donner naissance à des individus nouveaux. Il n'est pas impossible, d'autre part, qu'un fragment détaché d'un filament puisse se fixer à nouveau pour continuer en un autre point sa vie végétative. Cela expliquerait l'aspect de la figure 10 où l'on voit les premiers articles d'un filament présenter la division longitudinale qui ne s'observe d'habitude que dans la partie distale des individus ; à noter aussi que le cône de fixation y est remarquablement chromatophile. Il est vrai qu'une autre explication pourrait être fournie par une progression basipète de la division longitudinale plus rapide que l'accroissement du filament dû à la division transversale et qui lui permettrait ainsi d'atteindre jusqu'à la cellule initiale elle-même. 
Position systématique. - Tels sont les aspects divers que j'ai pu observer et qui se rapportent bien à un seul et même entophyte que je nommerai Anacamptothrix intestinalis n. g., n. sp.

Il n'y a pas de doute qu'il ne s'agisse d'un Schizophyte, mais il reste à en préciser les alfinités.

Il est un protophyte auquel on songe tout de suite, e'est celui qu'Engler a décrit en 1882 sous le nom de Beggiatoa multiseptata et pour qui il a créé l'année suivante le genre Phragmidiothrix. C'est une bactérie filamenteuse marine qu'il avait rencontré dans la baie de Kiel vivant en épiphyte sur Gammarus locusta sur qui il forme de petites taches blanches. Les filaments ont jusqu'à $100 \mu$ de longueur sur 3 à 12 de largeur; il y a une gaine très mince, à peine visible ; la cellule est d'abord cylindrique puis se replie ; elle présente une multiplication par « conidies » provenant par division dans les trois plans de cellules végétatives. Hansgirg (1891) n'accepta pas le genre Phragmidiothrix et le fit entrer dans le genre Crenothrix, mais en subdivisant toutefois ce genre en deux sections Phragmidiothrix pour les formes marines et Eucrenothrix pour les formes d'eau douce; pour lui, l'espèce d'Engler n'était pas différente. de celle qu'il avait lui-même établie en 1890 sous le nom de Crenothrix marina. Migula maintint toutefois en 1894 le genre d'Engler et précisa l'année suivante que, bien que, sans doute proche de Crenothrix marina, il valait mieux le conserver jusqu'à nouvelle étude. Celle-ci, malheureusement, n'a jamais été faite et si, par la suite un grand nombre d'auteurs (Chester, Kendall, Smith, Migula, etc...,) redonnent ce genre, ils ne font que reproduire la diagnose d'Engler. Quant au genre Crenothrix, ramené à l'unique espèce $C$. polyspora Cohn, il présente une gaine épaisse souvent brunie par l'oxyde de fer et des cellules cylindriques qui se divisent dans les trois plans pour donner des conidies (microconidies et macroconidies).

Notons que notre entophyte s'évase beaucoup moins que ces organismes, sa largeur dans les parties les plus âgées des filaments, celles qui contiennent les spores, ne dépassant guère 7 à $8 \mu$; qu'il présente une gaine mince, des cellules non cylindriques mais aplaties. Enfin, s'il présente des « conidies », et c'est peut-être là l'afffinité la plus frappante, nous avons vu que le mécanisme de formation en était différent et que la division des cellules végétatives ne se fait en réalité que suivant deux plans. La parenté qui, à première vue paraissait grande diminue à l'examen attentif.

D'autre part, les jeunes filaments évoquent irrésistiblement des affinités avec les Cyanophycées. On connait, parmi celles-ci, de 
nombreuses Oscillospiracées, dépourvues de pigment, adaptées à la vie parasitaire ou saprophytique ; mais elles ne se fixent pas, elles ne forment pas d'exospores, mais au contraire des endospores et là encore la parenté s'estompe.

L'absence d'ailleurs de documents cytologiques ne permet pas d'espérer actuellement préciser cette question; notre protiste ne pourra ainsi prendre qu'une place d'attente dans le groupe vague des entophytes filamenteux à statut encore indéterminé, si intéressants et cependant si mal connus.

\section{Bibliographie}

Buchanan (R. E.). $\rightarrow$ General Systematic Bacteriology. Baltimore, 1925, p. 423. ENGLER (A.). - Ueber die im Kieler-Hafen in dem sogenannten « tedten Grund» vorkommende Pilzformen. Verhandl. bot. Ver. Branderb., XXIV, 1882, p. 17.

- Ueber die Pilz-Vegetation des weissen oder todten Grundes in der Kieler Bucht. IV. Bericht der Comm. zur wissensch. Unters, der deutschen Meere in Kiel für 1877 bis 1881, 1883 Abt. I, p. 187.

Grassé (P. P.). - Anisomitus denisi n. g., n. sp., schizophyte de l'intestin du canard domestique. Ann. Parasitol. hum. et comp., III, 1925, p. 343.

Hansgirg (A.). - Ueber die Bacteriaceen-Gattung Phragmidiothrix Engler und einige Leptothrix-Arten. Bot. Zeit., XLIX, 1891, p. 313.

Langeron (M.). - Les Oscillariées parasites du tube digestif de l'homme et des animaux. Ann. Parasitol. hum. el comp., I, 1923, pp. 75 et 112.

Lavier (G.). - Sur quelques Flagellés intestinaux de poissons marins. Ann. de Parasitol. hum. et comp., XIV, 1936, p. 278.

Migula (W.). - Ueber ein neues System der Bakterien. Arb. bact. Inst. Karlsruhe, I, 1894 ; p. 235.

- Schyzomycetes in Engler et Prantl, Natürl. Pflanzen Famil., 1895, p. 20.

- System der Bakterien, II, Jena, Fischer, 1900, p. 1034.

- Allgemeine Morphologie, Entwickelungsgeschichte, Anatomie und Systematik der Schizomyceten in Lafar Handbuch der technischen Mykolo. gie, $2^{e}$ éd., 1904, p. 145.

Institut de Parasitologie de la Faculté de médecine de Paris (Directeur : Prof. E. Brumpt) et Laboratoire de Zoologie et Parasitologie de la Faculté de médecine de Lille (Directeur : Prof. G. Lavier). 